Article

\title{
Bifunctional Heterometallic Metal-Organic Frameworks for Solvent-Free Heterogeneous Cascade Catalysis
}

\author{
Mingming Zheng ${ }^{1,2, * \mathbb{D}}$, Yanxiang Wang ${ }^{2}$ and Pingyun Feng ${ }^{2, *}$ \\ 1 Oil Crops Research Institute, Chinese Academy of Agricultural Sciences, Hubei Key Laboratory of Lipid \\ Chemistry and Nutrition, Wuhan 430062, China \\ 2 Department of Chemistry, University of California, Riverside, CA 92521, USA; ywang146@ucr.edu \\ * Correspondence: zhengmingming@caas.cn (M.Z.); pyfeng@ucr.edu (P.F.)
}

Received: 17 February 2020; Accepted: 29 February 2020; Published: 8 March 2020

\begin{abstract}
A family of heterometallic metal-organic frameworks (MOFs) (CPM200s) harmoniously coexisting as Lewis acids and base (azo) sites were prepared. Seven CPM200s were employed as multifunctional heterogeneous cascade catalysts for the one-pot deacetalization-Knoevenagel reaction in a solvent-free system. Benefiting from the cooperation between Lewis acids from the open metal sites and base sites from the ligands, the CPM200s showed high activity and selectivity for the tandem reaction. The heterometallic 3D porous framework reported here not only offers a combination of two opposite active sites in the same framework of materials but also increases mass transfer of the substrate, thus maximizing the efficiency and substrate selectivity of the bifunctional catalysts. The CPM200s showed the highest turnover frequency (TOF), outperforming that of the reported MOFs in tandem with the deacetalization-Knoevenagel reaction. A strong correlation between the TOF and charge-to-radius ratio (z/r) of metal ions in the CPM200s was observed for the first time. The bifunctional CPM200s catalysts can be reused five times without significant loss of activity.
\end{abstract}

Keywords: heterometallic metal-organic frameworks; open metal sites; bifunctionalized catalyst; solvent-free; $3 \mathrm{D}$ porous; tandem reactions

\section{Introduction}

With the advantages of higher efficiency, less by-product, lower operating cost, and fewer purification steps, one-pot, multi-step cascading reactions are currently receiving considerable attention [1-5]. The applications of multi-functional catalysts in the one-pot reaction process not only simplify the operational procedure by omitting steps of the purification of the intermediate, but also provides a useful strategy for the development of new reactions that cannot be realized with other methods [6].

Among the different types of multifunctional catalysts, effective multifunctional heterogeneous catalysts have not been widely used in the multi-step cascading reactions, due to the challenge associated with the deactivation of different active sites, leaching of metal ions, and poor recyclability $[7,8]$. The introduction of multi-functional catalytic active sites, particularly both basic and acidic sites, on the same support has been considered a common strategy for developing heterogeneous catalysts for cascading reactions $[9,10]$. Thus far, a lot of acid-base multi-functional catalysts have been prepared [11,12], including mesoporous organosilicas [13-15], zeolite [16], cross-linked micelles [17], Pickering emulsions [18], and hyper-cross-linked nanospheres [19].

In accordance with the principles of "green chemistry," metal-organic frameworks (MOFs), which feature ordered frameworks, various metal nodes, large surface area, tunable pore sizes, and 
tailorable and accessible active sites, are actively used for heterogeneous catalysis [20-22]. With diverse functionalization capability on both organic ligands and inorganic nodes, MOFs could be a great candidate for bridging the gap between homogeneous and crystalline heterogeneous catalysts with high porosity and abundant active sites [23-25].

By tailoring the pore system in terms of shape and size of the pore, MOFs could introduce shape and size selectivity to the substrates [23,24]. For example, MIL-101 functionalized both with amino and sulfo groups [26] and JUC-199 with both open metal sites $\left(\mathrm{Zn}^{2+}\right)$ and basic sites $\left(-\mathrm{NH}_{2}\right)$ [3] was used to catalyze the one-pot deacatalization-Knoevenagel condensation in the presence of organic solvent. Nevertheless, the solvent-free tandem reaction catalyzed by MOFs was rarely evaluated.

MOFs with highly tunable inorganic building units especially with the easier integration of different metal cations into the same structure can be a great advantage for their applications [27-29] such as catalysis and gas adsorption and separation [30-32]. In addition to the flexible inorganic building units, organic ligands can also be controlled and tuned to further enhance the material properties. For example, MOFs containing both basic sites from the ligands part and Lewis acid sites from the metallic nodes possessing unsaturated open metal sites (OMSs) can potentially be integrated together. The synthesized MOFs could be used as bifunctional heterogeneous catalysts for one-pot tandem reactions [33-35]. Recently, a group of heterometallic MOFs $\left[\left(\mathrm{M}^{\mathrm{III}} \mathrm{M}^{\mathrm{II}}{ }_{2}(\mathrm{OH})(\mathrm{ABTC})_{1.5}\left(\mathrm{H}_{2} \mathrm{O}\right)_{3}\right)\right.$ $\left(\mathrm{ABTC}=\right.$ full form of this ligand) with clear mention of the trimeric $\left(\mathrm{M}^{\mathrm{II}}{ }_{2} \mathrm{M}^{\mathrm{III}}\left(\mu_{3}-\mathrm{OH}\right)\left(\mathrm{CO}_{2}\right)_{6}\right)$ clusters] with combinations of trivalent $\mathrm{M}^{\mathrm{III}}\left(\mathrm{Fe}^{3+}, \mathrm{V}^{3+}, \mathrm{Sc}^{3+}\right)$ and divalent $\mathrm{M}^{\mathrm{II}}\left(\mathrm{Mg}^{2+}, \mathrm{Mn}^{2+}, \mathrm{Co}^{2+}\right)$ cations was reported [30]. These metal centers on the network, especially unsaturated OMSs in the MOF, can act as active sites of catalysis [25]. Benefiting from the large surface area, three-dimensional (3D) porous framework, and abundant open metal sites, these CPM200s could potentially be used as heterogenous catalytic materials for different reactions [30].

Our objective in this research was to study and evaluate heterometallic MOFs as a multifunctional catalyst for the solvent-free tandem reaction. Benefiting from the cooperation between Lewis acids from the OMSs and the base sites from the ligands, the CPM200s exhibit a high activity and selectivity for the tandem deacetalization-Knoevenagel reaction. The heterometallic and 3D porous framework not only provides a combination of two complementary types of active sites, namely basic and acidic sites, but also enables fast contact of the substrates, thus maximizing the efficiency and selectivity. The discovery of a significant correlation between the turnover frequency (TOF) and the charge-to-radius ratio $(\mathrm{z} / \mathrm{r})$ of metal ions in MOFs was achieved for the first time.

\section{Results and Discussion}

\subsection{Catalyst Characterization}

The phase purity of prepared bulk samples including CPM200-Fe/Mg, $-\mathrm{V} / \mathrm{Mg},-\mathrm{Sc} / \mathrm{Mg},-\mathrm{Ga} / \mathrm{Mg}$, -In/Mg, -In/Co, and -In/Mn was proved by powder X-ray diffraction (PXRD) (Figure 1), which match with the simulated patterns, suggesting that the CPM200s were successfully prepared. The BET surface areas and pore volume of seven CPM200 MOFs were calculated from the $\mathrm{N}_{2}$ adsorption/desorption isotherm (Table S1). The BET surface areas and pore volumes of CPM200 MOFs varied in the ranges of $877-1459 \mathrm{~m}^{2} / \mathrm{g}$ and $0.43-0.72 \mathrm{~cm}^{3} / \mathrm{g}$, respectively [30]. The characteristic peak of the carbonyl stretching band $(\mathrm{C}=\mathrm{O})$ of $-\mathrm{COOH}$ at $1702 \mathrm{~cm}^{-1}$ was only found in the ligand (black) curve, implying that all carboxyl groups of ligands were deprotonated (Figure S1).

As shown by the thermogravimetric analysis (TGA) results (Figure S2), CPM200 MOFs experienced a slight weight loss of $5.2 \%-13.3 \%$ before $200{ }^{\circ} \mathrm{C}$, which proves the high framework stability.

In order to have a better understanding of these CPM-200 materials' catalytic activity, the Lewis acid and base property of the MOFs were examined using $\mathrm{NH}_{3}$ and $\mathrm{CO}_{2}$ temperature-programmed desorption (TPD), respectively. Figure S3 shows the $\mathrm{CO}_{2}$-TPD profiles of the CP200 (V/Mg), and the distribution of the Lewis basic sites was estimated following the guideline of Jiang et al. [31]. The Lewis basicity was considered weak in the $323-450 \mathrm{~K}$ range and medium to strong from 650 to 
900 K. According to Figure S3A, CP200 (V/Mg) showed a high population of strong Lewis basic sites, exhibiting TPD peaks around $820 \mathrm{~K}$. As shown in Figure S3B, the Lewis acidity of CP200 (V/Mg) can be classified into two groups from the patterns obtained: Medium (435 K) and strong (805 K).

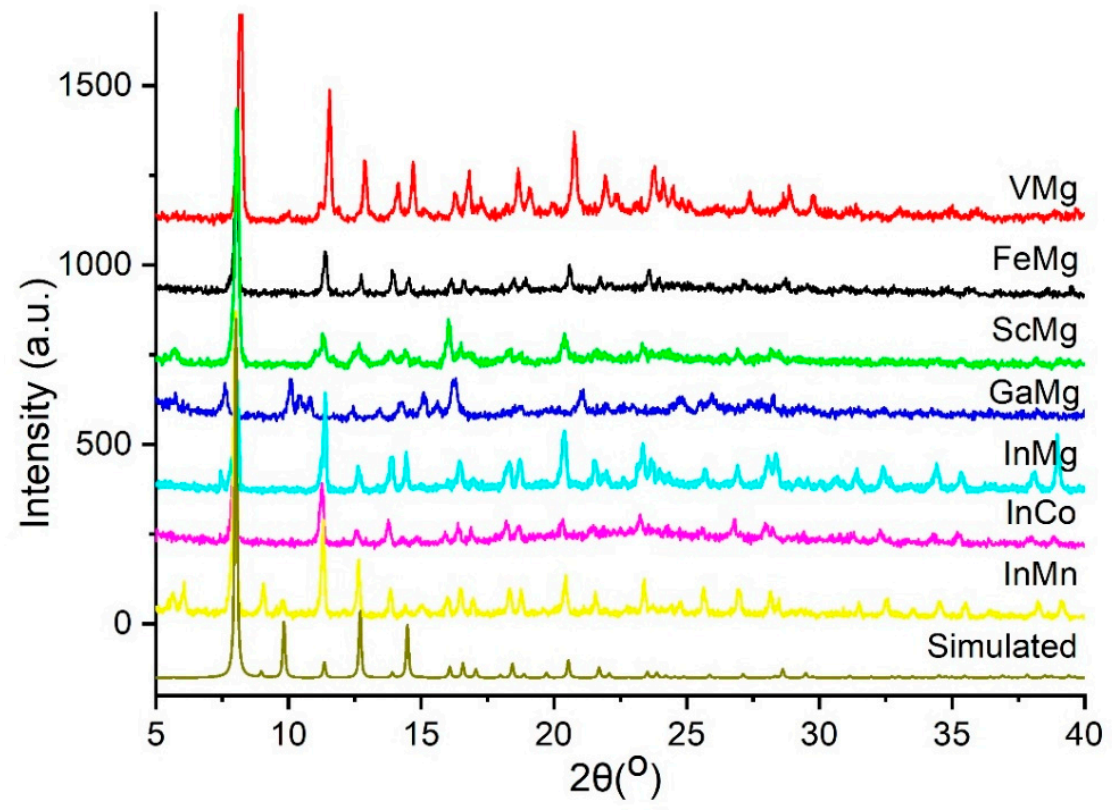

Figure 1. Experimental and simulated PXRD patterns for seven CPM200 metal-organic frameworks (MOFs).

\subsection{Catalytic One-Pot Deacetalization-Knoevenagel Condensation}

For the deacetalization-Knoevenagel condensation of benzaldehyde dimethylacetal with malononitrile, different solvents such as toluene, dioxane, acetonitrile, THF, DMF, and ethanol were used to identify the optimized reaction conditions (Table 1). Among these solvents, the conversion of the substrates in acetonitrile and THF were all below $23 \%$, while the reactions carried out in ethanol and DMF could reach above $90 \%$ conversion. When the reaction was carried out in toluene and dioxane, only about $5.5 \%-6.5 \%$ of conversion was obtained. From these results, it can be deduced that a higher conversion could be achieved in solvents with higher polarity, whereas it was more difficult to carry out the reaction in solvents with lower polarity [36]. However, the reaction under the solvent-free condition was impressively much faster than those in any solvent. The conversion rate reached $99 \%$ within $3 \mathrm{~h}$ in the solvent-free system. This could be mainly attributed to the direct interaction of the substrate with the open metal sites and basic ligands without competition from the solvent molecules. It is noteworthy that this environmentally friendly solvent-free system for the one-pot deacetalization-Knoevenagel condensation is reported here for the first time.

Table 1. Effect of solvents on one-pot deacetalization-Knoevenagel condensation reaction.

\begin{tabular}{cc}
\hline Solvent & Conversion (\%) \\
\hline Toluene & 6.5 \\
Dioxane & 5.5 \\
$\mathrm{CH}_{3} \mathrm{CN}$ & 15.9 \\
$\mathrm{THF}$ & 23.0 \\
$\mathrm{DMF}$ & 89.8 \\
EtOH & 90.2 \\
Solvent free & 99.4 \\
\hline
\end{tabular}


Up to now, only a few MOFs have been applied for the acid-base tandem reaction $[3,25]$. In the present work, CPM200s (Fe/Mg, V/Mg) could effectively catalyze benzaldehyde dimethylacetal (1) to form benzylidenemalononitrile (3) in almost quantitative yield in $3 \mathrm{~h}$ (Table 2, entry 1,2). On the contrary, $\mathrm{MgO}$ showed poor reactivity as a basic solid catalyst with a $23 \%$ yield of product 3 (entry 3). On the one hand, homogeneous catalysts including $\mathrm{HCl}$ and triethylamine were investigated for this reaction. The acid $(\mathrm{HCl})$ could only catalyze the deprotonation of 1 to benzaldehyde (2) (entry 4), which is similar to the previous reported result [3]. On the other hand, TEA and ZIF-8 as base catalysts could catalyze this reaction (the deprotonation of 1 to 2 ) with much lower yields (4.5\% and $3.2 \%$, respectively) and exhibit a higher yield for product $3(61.1 \%$ and $73.8 \%$, respectively). Nevertheless, a high content of byproducts from the self-condensation and oligomerization was found (entry 5,6 ) for the TEA and ZIF-8 catalytic systems. For the substrates with -Br and -OMe groups on the phenyl ring, the conversion rates were varied between $96.5 \%$ and $99 \%$ (entry 7-10) under the optimized conditions by using CPM200s as catalysts. The results proved that CPM200s possess wide adaptability, which could be used to catalyze a group of substitute molecules with similar structure in high yield. However, for the larger methylene substrate (cyanoacetic acid methyl ester), the conversion decreased sharply to below $13.7 \%$ under the same conditions. The results demonstrated that CPM200s can serve as bifunctional acid-base catalysts for the one-pot deacetalization-Knoevenagel condensation reaction with high activity.

Table 2. One-pot deacetalization-Knoevenagel condensation reaction with different catalysts.

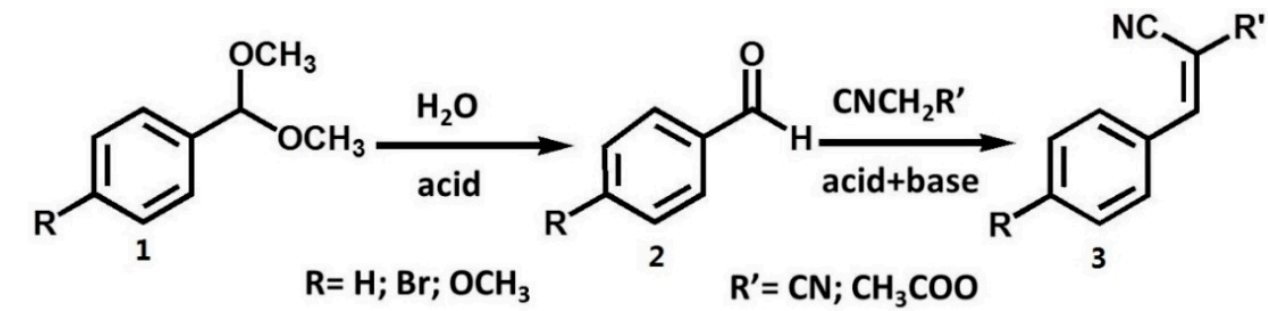

\begin{tabular}{cccccc}
\hline Entry & $\mathbf{R}$ & Catalyst & Conv. (\%) & Yield 2 (\%) $^{\mathbf{e}}$ & Yield 3 (\%) $^{\mathbf{e}}$ \\
\hline $1^{\mathrm{a}}$ & $\mathrm{H}$ & $\mathrm{Fe} / \mathrm{Mg}$ & 99.8 & 0.6 & 99.2 \\
2 & $\mathrm{H}$ & $\mathrm{V} / \mathrm{Mg}$ & 99.6 & 0.5 & 99.1 \\
$3^{\mathrm{b}}$ & $\mathrm{H}$ & $\mathrm{MgO}$ & 26.5 & 3.5 & 23.2 \\
$4^{\mathrm{c}}$ & $\mathrm{H}$ & $\mathrm{HCl}$ & 91.2 & 83.2 & 8.7 \\
$5^{\mathrm{c}}$ & $\mathrm{H}$ & $\mathrm{TEA}$ & 85.6 & 4.5 & 61.1 \\
$6^{\mathrm{c}}$ & $\mathrm{H}$ & $\mathrm{ZIF}-8$ & 90 & 3.2 & 73.8 \\
7 & $\mathrm{Br}$ & $\mathrm{V} / \mathrm{Mg}$ & 97.3 & 1.2 & 96.5 \\
8 & $\mathrm{Br}$ & $\mathrm{Fe} / \mathrm{Mg}$ & 98.4 & 1.4 & 97.2 \\
9 & $\mathrm{OCH} 3$ & $\mathrm{~V} / \mathrm{Mg}$ & 99.5 & 0.8 & 98.7 \\
10 & $\mathrm{OCH} 3$ & $\mathrm{Fe} / \mathrm{Mg}$ & 99.6 & 0.5 & 99.1 \\
$11^{\mathrm{d}}$ & $\mathrm{CH}_{3} \mathrm{COOCH}_{2} \mathrm{CN}$ & $\mathrm{Fe} / \mathrm{Mg}$ & 13.7 & 10.9 & 3.0 \\
$12^{\mathrm{d}}$ & $\mathrm{CH}_{3} \mathrm{COOCH}_{2} \mathrm{CN}$ & $\mathrm{V} / \mathrm{Mg}$ & 10.5 & 8.2 & 2.3 \\
\hline
\end{tabular}

a Reaction conditions: Substrate $(4 \mathrm{mmol})$, malononitrile $(6 \mathrm{mmol})$, catalyst $(0.04 \mathrm{mmol}), 80^{\circ} \mathrm{C}, 3 \mathrm{~h} .{ }^{\mathrm{b}} \mathrm{MgO}(0.2 \mathrm{mmol})$ was employed as the catalyst. ${ }^{\mathrm{c}} 0.1 \mathrm{mmol}$ of $\mathrm{HCl}$, triethylamine (TEA), or ZIF-8 was used as the catalyst. ${ }^{\mathrm{d}}$ Methyl cyanoacetate was used as the methylene substrate instead of malononitrile. ${ }^{\text {e }}$ The yields were determined by GC-FID.

\subsection{Selectivity of Substrates}

In order to further prove whether the tandem reaction occurred on the surface or inside the pores of the CPM200s, active aldehyde and methylene substrates with different dimensions were selected for the Knoevenagel reaction (Figure 2). For malononitrile, the conversion of benzaldehyde was above $96.5 \%$ in $4 \mathrm{~h}$ with seven CPM200s as the catalysts. As for larger aldehydes such as heptanal and 4-tert-butyl-benzyldehyde, the condensation reaction with malononitrile $(4.5 \times 2.2 \AA)$ showed an apparent decline with the conversion varying from $72.6 \%$ to $88.4 \%$ and $17.4 \%$ to $48.9 \%$, respectively. As for the larger methylene substrate such as methyl cyanoacetate $(4.8 \times 4.7 \AA)$, the condensation with 
benzaldehyde sharply decreased to below $5 \%$ under the same conditions. Catalysis efficiency depends on the interactions between the reactant and the active sites of the catalyst. This result may be ascribed to the mass transfer limitation of large-sized substitutes into the 3D porous framework. By contrast, the smaller molecules are easily accommodated into the channels and activated by the open metal sites and basic azo ligands. As the cage size of CPM200s is $12.8 \times 12.8 \AA$ with a window size of $7.6 \times 9.5 \AA$, larger substrates were excluded by the porous CPM200s, which indicates that the reactions occur inside the 3D porous framework. The CPM200s show excellent molecular size selectivity toward both aldehyde and methylene substrates.

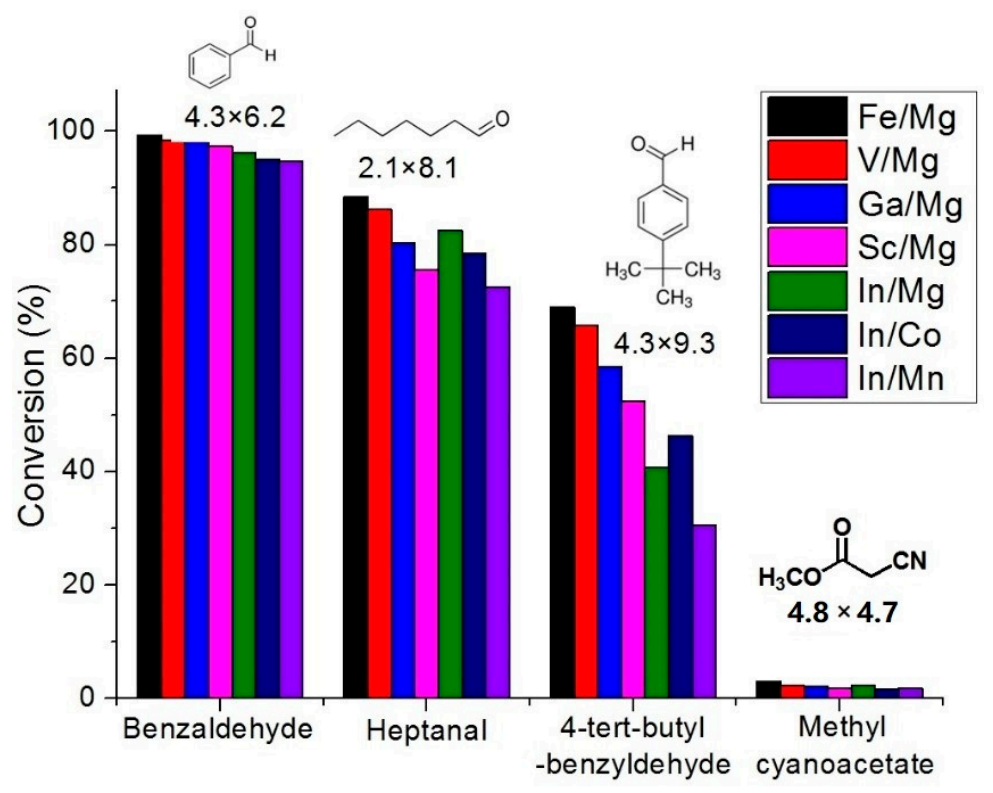

Figure 2. Size selectivity of CPM200 MOF catalysts with different aldehydes.

\subsection{Reaction Mechanism for the One-Pot Tandem Reaction}

The tandem reaction took place in two steps. The time course of substrates and products during the reaction is shown in Figure 3. The result proves that benzylidene malononitrile was far more easily generated than the deacetalization of benzaldehyde dimethylacetal. The concentration of the intermediate product (benzaldehyde) increased to reach a maximum in $0.5 \mathrm{~h}$ initially. Then, the generation of benzylidene malononitrile became the major reaction path and the whole reaction was finished in $3 \mathrm{~h}$. The final product and intermediate distribution showed a typical variation trend of one-pot tandem reactions [25].

In order to further study the roles of metal ions in one-pot tandem reaction catalysis, the relationship between $\mathrm{z} / \mathrm{r}$ and the turnover frequency (TOF) was investigated. As shown in Figure 4, when $\mathrm{In}^{3+}$ was fixed, the catalysis activity (related to TOF) of CPM200-In/ $\mathrm{M}^{\mathrm{II}}$ was confirmed to the order of $\mathrm{Mg}$ $>\mathrm{Co}>\mathrm{Mn}$. If $\mathrm{Mg}^{2+}$ was settled, the catalysis activity of CPM200-M ${ }^{\mathrm{III}} / \mathrm{Mg}$ respected the order of $\mathrm{Fe} \approx \mathrm{V}>\mathrm{Ga}>\mathrm{Sc}>\mathrm{In}$ (Figure $4 \mathrm{~b}$ ). As the same basic ligand is used in the reactions, the catalysis activity may be ascribed to the interactions between substrates and Lewis acid sites, which are the open metal sites generated by dehydration of the trimeric $\left(\mathrm{M}^{\mathrm{II}}{ }_{2} \mathrm{M}^{\mathrm{III}}\left(\mu_{3}-\mathrm{OH}\right)\left(\mathrm{CO}_{2}\right)_{6}\left(\mathrm{H}_{2} \mathrm{O}\right)_{3}\right)$ clusters in the CPM200s to give $\left(\mathrm{M}_{2}^{\mathrm{II}} \mathrm{M}^{\mathrm{III}}\left(\mu_{3}-\mathrm{OH}\right)\left(\mathrm{CO}_{2}\right)_{6}\right)$ [27-29]. The parameters of $\mathrm{z} / \mathrm{r}$ have a significant index of electrostatic contribution to the bond energy and are related to the role open metal sites play in the catalysis properties. Until now, no relationship between $\mathrm{z} / \mathrm{r}$ and TOF has been reported for materials in the application of catalysis. Here, a strong relationship between $\mathrm{z} / \mathrm{r}$ and TOF for the one-pot tandem deacetalization-Knoevenagel condensation reaction was observed for the first time. 


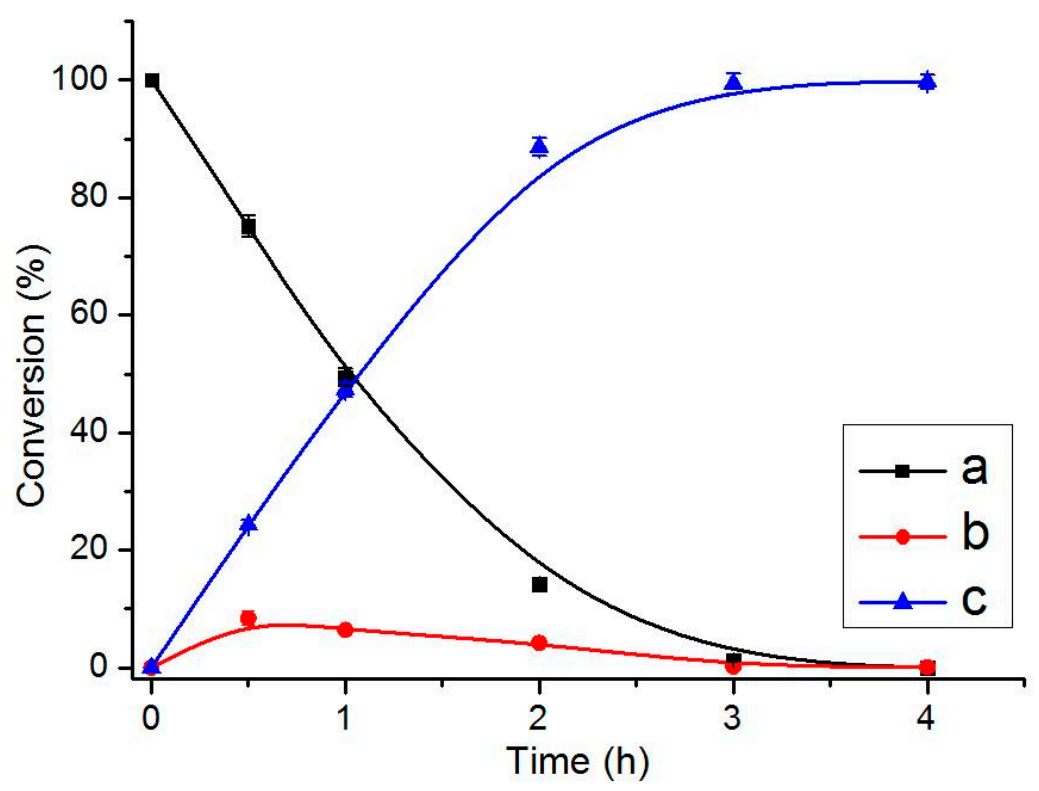

Figure 3. Results of the catalytic activity of the CPM200 Fe/Mg catalyst for the deacetalizationKnoevenagel condensation tandem reaction. Conversion versus reaction time of (a) dimethylacetal, (b) benzaldehyde, and (c) benzylidenemalononitrile.

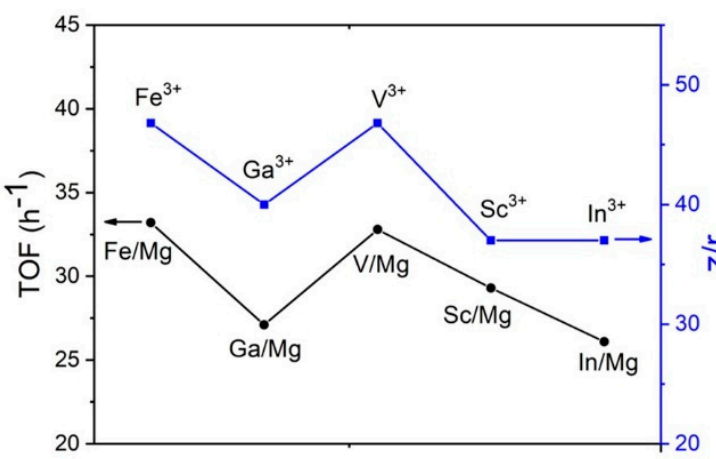

(a)

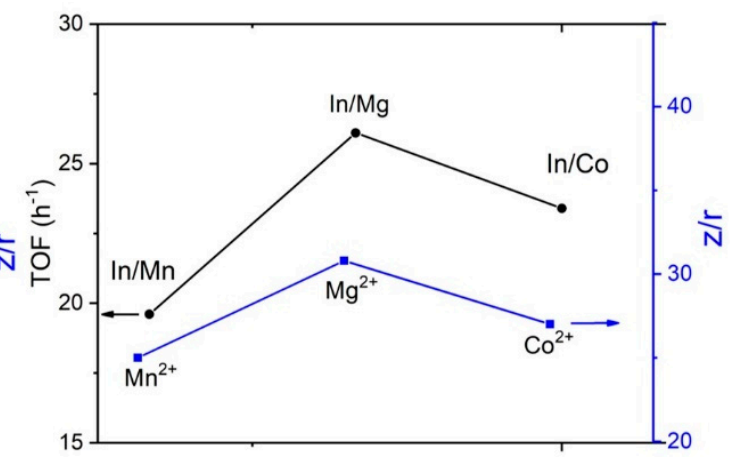

(b)

Figure 4. Correlation between catalysis activity (turnover frequency (TOF)) and charge-to-radius ratio (z/r) of metal ions for CPM200s. (a) CPM200-M"III/Mg; (b) CPM200-In/M" .

Normally, the Knoevenagel condensation is catalyzed by a solid base such as amine-functionalized solid supports [36,37]. In this study, although the seven CPM200s possess the same basic ligand with azo groups, the performance in the solvent-free Knoevenagel condensation catalysis was different from each other. As shown in Figure S5, the TOF values were $196.8(\mathrm{Fe} / \mathrm{Mg}), 190.6(\mathrm{~V} / \mathrm{Mg}), 181.0(\mathrm{Sc} / \mathrm{Mg})$, $151.6(\mathrm{Ga} / \mathrm{Mg}), 144.6(\mathrm{In} / \mathrm{Mg}), 121.2(\mathrm{In} / \mathrm{Co})$, and $100.4 \mathrm{~h}^{-1}(\mathrm{In} / \mathrm{Mn})$, respectively, which are much higher than those of other MOFs [38,39]. As the activity order matches well with the $\mathrm{z} / \mathrm{r}$ order of the metal cations, such diverse catalysis activity could be associated with the different strength of Lewis acid sites generated from the unsaturated metal cations on the framework. A mechanism for the one-pot tandem reaction between benzaldehyde dimethylacetal with malononitrile is proposed (Figure 5). More specifically, the Lewis acid sites could interact with the carbonyl oxygen of benzaldehyde; thus, the $\mathrm{C}=\mathrm{O}$ bond is more polarized to promote the nucleophilic attack at the $\mathrm{C}$ atom; the base sites would lead to the deprotonation of the methylene group, producing the carbanion that attacks the carbonyl $\mathrm{C}$ atom of benzaldehyde. The presence of both acid and base sites on the same framework could explain the outstanding property of CPM200s in the Knoevenagel reaction. The bifunctional character has also been used to explain the activity of the UiO66- $\mathrm{NH}_{2}$ for the Knoevenagel condensation [37]. These results 
prove the functionality of the different active sites: The Lewis acid sites catalyze deacetalization and the Lewis acids together with base sites synergistically catalyze Knoevenagel condensation.

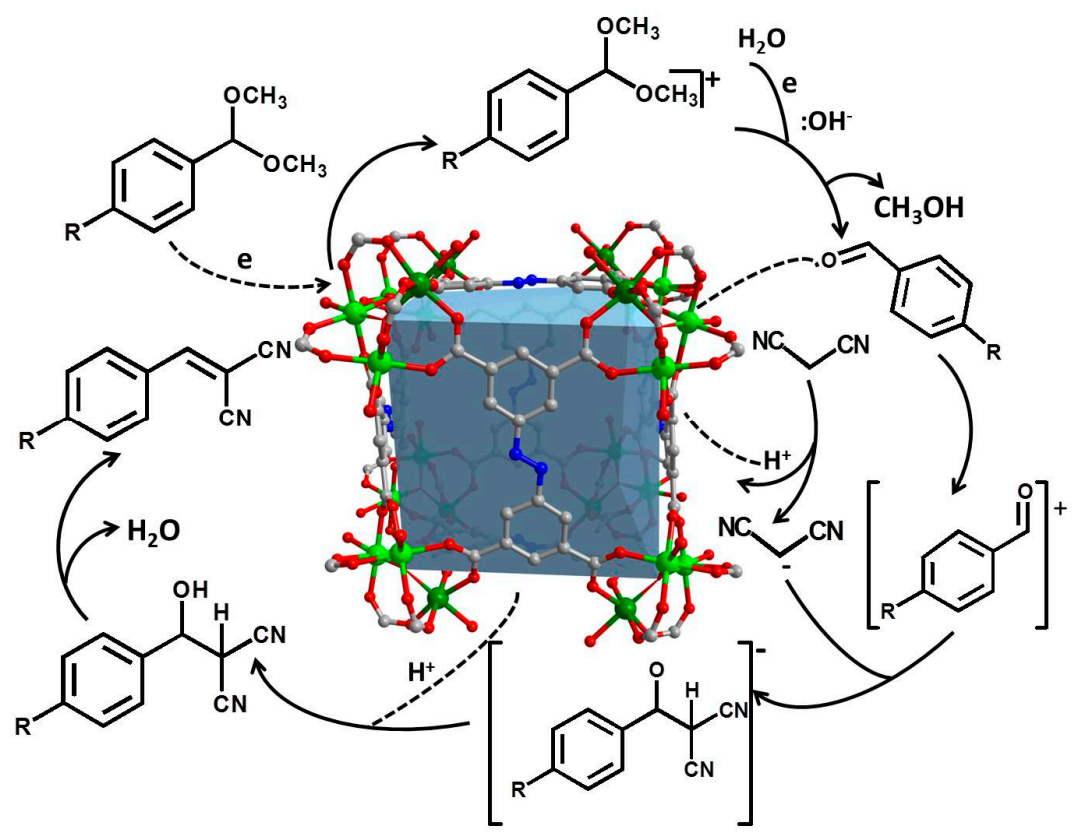

Figure 5. Mechanism for CPM200s-catalyzed tandem deacetalization-Knoevenagel condensation.

\subsection{Stability and Recyclability of the CPM200s Catalyst}

The recyclability of the CPM200s was investigated to evaluate the applicability and stability of the bifunctional catalyst. The reaction used benzaldehyde and malononitrile as the substrates in the presence of CPM200s Fe/Mg, V/Mg, Sc/Mg, and $\mathrm{In} / \mathrm{Mg}$ under the optimal reaction conditions. The recycled catalysts exhibited almost no loss of activity over five runs (Figure 6); the conversion of the targeted 2-benzylidenemalononitrile product remained over $95 \%$ after five runs.

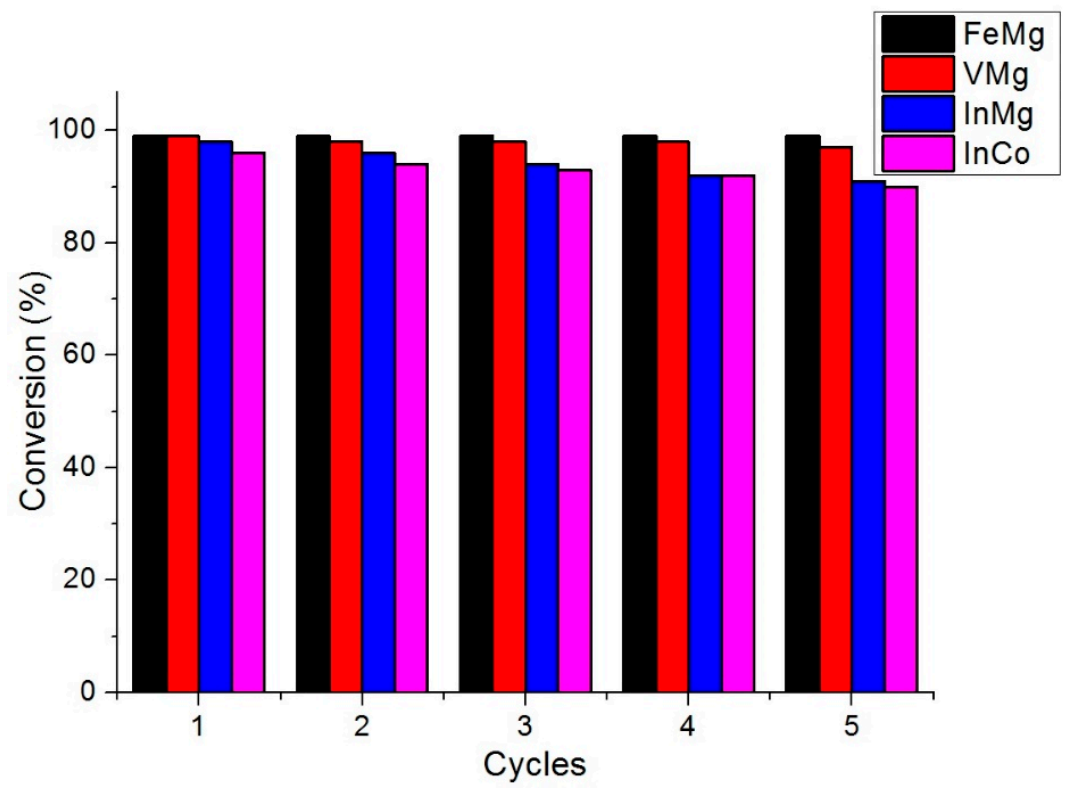

Figure 6. Recycle tests of four CPM200s for the tandem deacetalization-Knoevenagel reaction.

To further study the possibility of catalyst leaching of CPM200s during the one-pot tandem reaction, the catalyst was separated after $1 \mathrm{~h}$ of reaction (ca. $48 \%$ yield), and the reaction solution was 
further reacted for $3 \mathrm{~h}$. As shown in Figure 7, the yield of benzyl alcohol remained unchanged after separation of the catalyst. This result indicates the stable covalently bonded structure of the CPM200s and that there was no leakage of the metal or 3,3',5,5'-azobenzenetetracarboxylic acid $\left(\mathrm{H}_{4} \mathrm{ABTC}\right)$ ligand [19]. Powder X-ray characterization of the reused CPM200s was carried out on fresh and recycled catalysts, showing almost similar PXRD (Figure S4) profiles. Thus, there is no structural alteration of the CPM200s after it is recycled and reused.

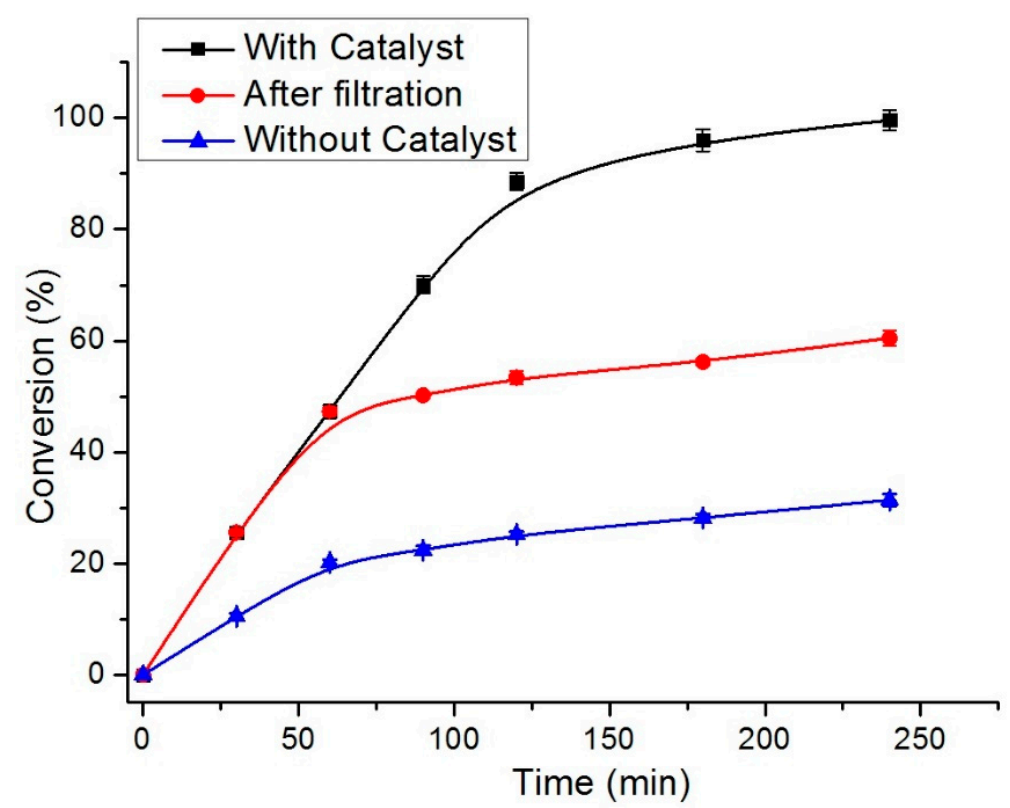

Figure 7. Filtration test for the tandem deacetalization-Knoevenagel reaction over CPM200 Fe/Mg, compared to the reactions with and without the catalyst.

\section{Experimental Section}

\subsection{Instrumentation and Materials}

IR spectra were recorded on an EQUINOX 55 FT-IR spectrometer (Bruker, Karlsruhe, German) using $\mathrm{KBr}$ pellets technology. Powder X-ray diffraction (XRD) data were collected on a D8 Advance powder diffraction meter (Bruker, Karlsruhe, German) with $\mathrm{CuK} \alpha$ radiation $(40 \mathrm{kV}, 40 \mathrm{~mA}, \lambda=1.5418 \AA$ ). TGA was performed by heating from room temperature to $900{ }^{\circ} \mathrm{C}$ at a rate of $10^{\circ} \mathrm{C} \mathrm{min}-1$ under a flow of nitrogen. The structure of products was confirmed by a GCT premier GC-MS (Waters, Milford, USA). The conversions of the substrates were examined on a GC 2010 (Shimadzu, Kyoto, Japan).

\subsection{General Procedure for CPM200s Preparation}

The CPM200s were prepared using our reported method [30]. All the as-synthesized CPM200 samples were immersed in $\mathrm{CH}_{2} \mathrm{Cl}_{2}$ for 3 days; during the exchange, the $\mathrm{CH}_{2} \mathrm{Cl}_{2}$ was refreshed three times. The resulting $\mathrm{CH}_{2} \mathrm{Cl}_{2}$-exchanged samples were then evacuated in a vacuum drying oven at $80^{\circ} \mathrm{C}$ for $12 \mathrm{~h}$.

\subsection{General Procedure for the One-Pot Deacetalization-Knoevenagel Reaction}

In a typical experiment, the catalytic reactions were carried out in a $5 \mathrm{~mL}$ glass reactor vial. A mixture of substrate $(4 \mathrm{mmol})$, malononitrile $(6 \mathrm{mmol})$, and $30 \mathrm{mg}$ of the catalyst was stirred at $500 \mathrm{rpm}$ at $80^{\circ} \mathrm{C}$ for a certain time. A portion of the reaction mixture $(10 \mu \mathrm{L})$ was periodically removed from the system and analyzed by a Shimadzu GC-2010 to determine the conversion of substrates and yield of products. 


\subsection{General Procedure for Recycling of the CPM200s}

To test the recyclability of the CPM200s, a mixture of the reaction system was filtered at the end of the reaction. The obtained solid catalyst was washed with ethanol $(4.0 \mathrm{~mL} \times 3)$ and then $\mathrm{CH}_{2} \mathrm{Cl}_{2}$ $(4.0 \mathrm{~mL} \times 1)$. After $12 \mathrm{~h}$ of drying under vacuum at $80^{\circ} \mathrm{C}$, the catalyst was subjected to the next run.

\section{Conclusions}

A family of heterometallic MOFs with a 3D porous framework and a high density of OMSs and base sites was prepared. The synergistic effects of OMSs and azo ligands bestowed CPM200s with the highest TOF among known MOFs and placed them in striking distance from the record so far for the tandem deacetalization-Knoevenagel reaction in a solvent-free system (Table 3). The CPM200s catalysts exhibited the highest activity and outstanding recyclability. Moreover, with different combinations of metal ions in the same MOF, a strong correlation between TOF and charge-to-radius ratio has been established for the first time. This study demonstrates that heterometallic MOFs with great versatility can be used as excellent multi-functional catalytic materials for organic reactions.

Table 3. Summary of catalysis property for bifunctional MOFs.

\begin{tabular}{|c|c|c|c|c|c|}
\hline MOFs & Conversion & Time (h) & Catalyst Amount (\%) & TOF $\left(h^{-1}\right)$ & Ref. \\
\hline CPM200-VMg & 98.5 & & & 32.8 & \\
\hline CPM200-FeMg & 99.6 & & & 33.2 & \\
\hline CPM200-GaMg & 81.4 & & & 27.1 & \\
\hline CPM200-ScMg & 88.0 & 3 & $1 \%$ & 29.3 & This work \\
\hline CPM200-InMg & 78.3 & & & 26.1 & \\
\hline CPM200-InCo & 70.2 & & & 23.4 & \\
\hline CPM200-InMn & 58.9 & & & 19.6 & \\
\hline $\mathrm{J}-199$ & 99 & 4 & $6 \%$ & 4.1 & [3] \\
\hline MIL-101-AB-x & 99 & 2 & $4 \%$ & 12.5 & [26] \\
\hline
\end{tabular}

Supplementary Materials: The following are available online at http://www.mdpi.com/2073-4344/10/3/309/s1, Figure S1: FT-IR spectra for ligand and seven $\mathrm{CH}_{2} \mathrm{Cl}_{2}$-exchanged CPM200 MOFs; Figure S2: TGA curves for $\mathrm{CH}_{2} \mathrm{Cl}_{2}$-exchanged CPM200 MOFs; Figure S3: $\mathrm{CO}_{2}$-TPD (A) and $\mathrm{NH}_{3}$-TPD (B) profiles of CPM200 V/Mg catalysts; Figure S4: Experimental PXRD patterns before and after used and simulated PXRD patterns for CPM-200 MOFs, Table S1: Summary of molecular weight, surface area and pore volume data for CPM-200 MOFs.

Author Contributions: M.Z. contributed to data curation, investigation and writing-original draft; Y.W. contributed to formal analysis and methodology; P.F. helped with experiment design, final version improvement and project administration. All authors have read and agreed to the published version of the manuscript.

Funding: The research was funded by the US Department of Energy, Office of Basic Energy Sciences, Materials Sciences and Engineering Division under Award No. DE-SC0010596 (P.F.).

Acknowledgments: M.Z. would like to thank the support from the Program of China Scholarships Council (No. 201603250035).

Conflicts of Interest: The author declares no conflict of interest.

\section{References}

1. Miyamura, H.; Kobayashi, S. Tandem oxidative processes catalyzed by polymer-incarcerated multimetallic nanoclusters with molecular oxygen. Acc. Chem. Res. 2014, 47, 1054-1066. [CrossRef] [PubMed]

2. Raja, R.; Potter, M.E.; Newland, S.H. Predictive design of engineered multifunctional solid catalysts. Chem. Commun. 2014, 50, 5940-5957. [CrossRef] [PubMed]

3. He, H.; Sun, F.; Aguila, B.; Perman, J.A.; Ma, S.; Zhu, G. A bifunctional metal-organic framework featuring the combination of open metal sites and Lewis basic sites for selective gas adsorption and heterogeneous cascade catalysis. J. Mater. Chem. A 2016, 4, 15240-15246. [CrossRef]

4. Lee, J.M.; Na, Y.; Han, H.; Chang, S. Cooperative multi-catalyst systems for one-pot organic transformations. Chem. Soc. Rev. 2004, 33, 302-312. [CrossRef] 
5. Wasilke, J.-C.; Obrey, S.J.; Baker, R.T.; Bazan, G.C. Concurrent tandem catalysis. Chem. Rev. 2005, 105, 1001-1020. [CrossRef]

6. Choo, G.C.; Miyamura, H.; Kobayashi, S. Synergistic cascade catalysis by metal nanoparticles and Lewis acids in hydrogen autotransfer. Chem. Sci. 2015, 6, 1719-1727. [CrossRef]

7. Voit, B. Sequential One-Pot Reactions Using the Concept of “Site Isolation”. Angew.Chem. Int. Ed. 2006, 45, 4238-4240. [CrossRef]

8. Gladysz, J.A. Introduction: Recoverable catalysts and reagents perspective and prospective. J. Am. Chem. Soc. 2002, 102, 3215-3216.

9. Margelefsky, E.L.; Zeidan, R.K.; Davis, M.E. Cooperative catalysis by silica-supported organic functional groups. Chem. Soc. Rev. 2008, 37, 1118-1126. [CrossRef]

10. Motokura, K.; Tada, M.; Iwasawa, Y. Heterogeneous organic base-catalyzed reactions enhanced by acid supports. J. Am. Chem. Soc. 2007, 129, 9540-9541. [CrossRef]

11. Zhang, F.; Jiang, H.; Wu, X.; Mao, Z.; Li, H. Organoamine-functionalized graphene oxide as a bifunctional carbocatalyst with remarkable acceleration in a one-pot multistep reaction. ACS Appl. Mater. Interfaces 2015, 7, 1669-1677. [CrossRef] [PubMed]

12. Zhang, F.; Jiang, H.; Li, X.; Wu, X.; Li, H. Amine-functionalized GO as an active and reusable acid-base bifunctional catalyst for one-pot cascade reactions. ACS Catal. 2014, 4, 394-401. [CrossRef]

13. Shylesh, S.; Wagner, A.; Seifert, A.; Ernst, S.; Thiel, W.R. Cooperative Acid-Base Effects with Functionalized Mesoporous Silica Nanoparticles: Applications in Carbon-Carbon Bond-Formation Reactions. Chem. Eur. J. 2009, 15, 7052-7062. [CrossRef] [PubMed]

14. Gao, J.; Zhang, X.; Lu, Y.; Liu, S.; Liu, J. Selective functionalization of hollow nanospheres with acid and base groups for cascade reactions. Chem. Eur. J. 2015, 21, 7403-7407. [CrossRef] [PubMed]

15. Shylesh, S.; Wagener, A.; Seifert, A.; Ernst, S.; Thiel, W.R. Mesoporous organosilicas with acidic frameworks and basic sites in the pores: An approach to cooperative catalytic reactions. Angew.Chem. Int. Ed. 2010, 49, 184-187. [CrossRef]

16. Liu, B.; Wattanaprayoon, C.; Oh, S.C.; Emdadi, L.; Liu, D. Synthesis of organic pillared MFI zeolite as bifunctional acid-base catalyst. Chem. Mater. 2015, 27, 1479-1487. [CrossRef]

17. Lee, L.-C.; Lu, J.; Weck, M.; Jones, C.W. Acid-base bifunctional shell cross-linked micelle nanoreactor for one-pot tandem reaction. ACS Catal. 2016, 6, 784-787. [CrossRef]

18. Yang, H.; Fu, L.; Wei, L.; Liang, J.; Binks, B.P. Compartmentalization of incompatible reagents within pickering emulsion droplets for one-pot cascade reactions. J. Am. Chem. Soc. 2015, 137, 1362-1371. [CrossRef]

19. Jia, Z.; Wang, K.; Tan, B.; Gu, Y. Hollow hyper-cross-linked nanospheres with acid and base sites as efficient and water-stable catalysts for one-pot tandem reactions. ACS Catal. 2017, 7, 3693-3702. [CrossRef]

20. Chughtai, A.H.; Ahmad, N.; Younus, H.A.; Laypkov, A.; Verpoort, F. Metal-organic frameworks: Versatile heterogeneous catalysts for efficient catalytic organic transformations. Chem. Soc. Rev. 2015, 44, 6804-6849. [CrossRef]

21. Furukawa, H.; Cordova, K.E.; O'Keeffe, M.; Yaghi, O.M. The chemistry and applications of metal-organic frameworks. Science 2013, 341, 1230444. [CrossRef] [PubMed]

22. Zhao, M.; Ou, S.; Wu, C.-D. Porous metal-organic frameworks for heterogeneous biomimetic catalysis. Acc. Chem. Res. 2014, 47, 1199-1207. [CrossRef] [PubMed]

23. Zhao, S.-N.; Song, X.-Z.; Song, S.-Y.; Zhang, H.-j. Highly efficient heterogeneous catalytic materials derived from metal-organic framework supports/precursors. Coord. Chem. Rev. 2017, 337, 80-96. [CrossRef]

24. Wu, C.D.; Zhao, M. Incorporation of molecular catalysts in metal-organic frameworks for highly efficient heterogeneous catalysis. Adv. Mater. 2017, 29, 1605446. [CrossRef]

25. García-García, P.; Müller, M.; Corma, A. MOF catalysis in relation to their homogeneous counterparts and conventional solid catalysts. Chem. Sci. 2014, 5, 2979-3007. [CrossRef]

26. Liu, H.; Xi, F.-G.; Sun, W.; Yang, N.-N.; Gao, E.-Q. Amino-and sulfo-bifunctionalized metal-organic frameworks: One-pot tandem catalysis and the catalytic sites. Inorg. Chem. 2016, 55, 5753-5755. [CrossRef]

27. Zheng, S.-T.; Wu, T.; Chou, C.; Fuhr, A.; Feng, P.; Bu, X. Development of composite inorganic building blocks for MOFs. J. Am. Chem. Soc. 2012, 134, 4517-4520. [CrossRef]

28. Zheng, S.-T.; Zhao, X.; Lau, S.; Fuhr, A.; Feng, P.; Bu, X. Entrapment of metal clusters in metal-organic framework channels by extended hooks anchored at open metal sites. J. Am. Chem. Soc. 2013, 135, 10270-10273. [CrossRef] 
29. Sajna, K.; Fracaroli, A.M.; Yaghi, O.M.; Tashiro, K. Modular Synthesis of Metal-Organic Complex Arrays Containing Precisely Designed Metal Sequences. Inorg. Chem. 2015, 54, 1197-1199. [CrossRef]

30. Zhai, Q.-G.; Bu, X.; Mao, C.; Zhao, X.; Feng, P. Systematic and dramatic tuning on gas sorption performance in heterometallic metal-organic frameworks. J. Am. Chem. Soc. 2016, 138, 2524-2527. [CrossRef]

31. Jiang, G.; Zhang, L.; Zhao, Z.; Zhou, X.; Duan, A.; Xu, C.; Gao, J. Highly effective P-modified HZSM-5 catalyst for the cracking of C4 alkanes to produce light olefins. Appl. Catal. A Gen. 2008, 340, 176-182. [CrossRef]

32. Mukherjee, S.; Desai, A.V.; Ghosh, S.K. Potential of metal-organic frameworks for adsorptive separation of industrially and environmentally relevant liquid mixtures. Coord. Chem. Rev. 2018, 367, 82-126. [CrossRef]

33. Dau, P.V.; Cohen, S.M. A bifunctional, site-isolated metal-organic framework-based tandem catalyst. Inorg. Chem. 2015, 54, 3134-3138. [CrossRef] [PubMed]

34. Prasad, R.R.; Dawson, D.M.; Cox, P.A.; Ashbrook, S.E.; Wright, P.A.; Clarke, M.L. A Bifunctional MOF Catalyst Containing Metal-Phosphine and Lewis Acidic Active Sites. Chem. Eur. J. 2018, 24, 15309-15318. [CrossRef] [PubMed]

35. Yang, D.; Gates, B.C. Catalysis by metal organic frameworks: Perspective and suggestions for future research. ACS Catal. 2019, 9, 1779-1798. [CrossRef]

36. Beheshti, S.; Morsali, A. Post-synthetic cation exchange in anionic metal-organic frameworks; a novel strategy for increasing the catalytic activity in solvent-free condensation reactions. RSC Adv. 2014, 4, 41825-41830. [CrossRef]

37. Luan, Y.; Qi, Y.; Gao, H.; Andriamitantsoa, R.S.; Zheng, N.; Wang, G. A general post-synthetic modification approach of amino-tagged metal-organic frameworks to access efficient catalysts for the Knoevenagel condensation reaction. Mater. Chem. A 2015, 3, 17320-17331. [CrossRef]

38. Wang, X.-S.; Liang, J.; Li, L.; Lin, Z.-J.; Bag, P.P.; Gao, S.-Y.; Huang, Y.-B.; Cao, R. An anion metal-organic framework with Lewis basic sites-rich toward charge-exclusive cationic dyes separation and size-selective catalytic reaction. Inorg. Chem. 2016, 55, 2641-2649. [CrossRef]

39. Almáši, M.; Zeleňák, V.; Opanasenko, M.; Čejka, J. A novel nickel metal-organic framework with fluorite-like structure: Gas adsorption properties and catalytic activity in Knoevenagel condensation. Dalton Trans. 2014, 43, 3730-3738. [CrossRef] 